\title{
Webizing Collaborative Interaction Space for Cross Reality with Various Human Interface Devices
}

\author{
Daeil Seo \\ Korea Institute of Science and Technology \\ xdesktop@kist.re.kr
}

\author{
Byounghyun Yoo \\ Korea Institute of Science and Technology \\ yoo@byoo.net
}

\author{
Heedong Ko \\ Korea Institute of Science and Technology \\ ko@kist.re.kr
}

\begin{abstract}
Recently, web based cross reality (XR) concept embracing virtual reality (VR), augmented reality (AR), and mixed reality (MR) technology has emerged, but user interaction devices for collaborative work supported in web environments are limited. The Virtual-Reality Peripheral Network (VRPN) in the traditional VR environment provides a device-independent and networktransparent interface. To promote the development of web based $\mathrm{XR}$ applications, a common method is required to support a collaborative interaction space in $\mathrm{VR}, \mathrm{AR}$, and $\mathrm{MR}$ contexts according to the user environment and various interaction devices that can be used without limitation of XR content in web based XR environments as well. In this paper, we propose a webizing method for a collaborative interaction space that provides user authentication and manages user sessions in XR environments. In addition, the webizing method supports human interface devices and related events via an interaction adaptor that delivers events based on user session and converts event messages according to XR content types such as VRPN messages, X3D sensor data, and HTML DOM events to deal with interaction events.
\end{abstract}

\section{CCS CONCEPTS}

- Human-centered computing $\rightarrow$ Interaction paradigms; Web-based interaction; Collaborative interaction; Mixed / augmented reality; Virtual reality

\section{KEYWORDS}

Webizing, collaboration, human interface devices, user interaction, cross reality

ACM Reference format:

Daeil Seo, Byounghyun Yoo, and Heedong Ko. 2018. Webizing Collaborative Interaction Space for Cross Reality with Various Human Interface Devices. In Proceedings of 23rd International Conference on Web3D Technology, Poznań, Poland, June 2018 (Web3D '18), 8 pages.

DOI: $10.1145 / 3208806.3208808$

Permission to make digital or hard copies of part or all of this work for personal or classroom use is granted without fee provided that copies are not made or distributed for profit or commercial advantage and that copies bear this notice and the full citation on the first page. Copyrights for third-party components of this work must be honored. For all other uses, contact the Owner/Author.

Web3D '18, June 20-22, 2018, Poznan, Poland

(C) 2018 Copyright is held by the owner/author(s)

ACM ISBN 978-1-4503-5800-2/18/06.

https://doi.org/10.1145/3208806.3208808

\section{INTRODUCTION}

Recently, cross reality (XR) has been used to represent another way to consider the reality continuum [Somasegar 2017; Wikipedia 2017]. XR technology facilitating cross-platform standard eliminating industry fragmentation by enabling applications to be written once to run on any XR system, and to access XR devices has been introduced and now people can easily access XR technology. With the spread of XR technology, a form of head-mounted display (HMD), mobile virtual reality (VR) devices such as Google Cardboard, Samsung Gear VR, and Oculus Rift as well as mobile augmented reality (AR) on smartphones have been widely distributed. In addition, motion controller devices for user interaction in XR environments have also increased. At the beginning of XR technology development, $\mathrm{XR}$ content was not easy to distribute because it was created for a specific applications and interaction devices and the barrier in the way of this was high. Dionisio et al. [2013] pointed out that interoperability, scalability, ubiquity, and realism are important for a fully-realized 3D virtual world.

The web environment already provides the latest updated content using web browsers with zero-overhead installations, updates, and maintenance and also enables full 3D rendering using WebGL without third-party plugins. Declarative 3D content languages such as Virtual Reality Modeling Language (VRML) [VRML Consortium 1997], eXtensible 3D (X3D) [X3D 2004], and A-Frame [Mozilla 2015] provide common representations to create XR content on the web. To access VR devices on the Web, WebVR provides interfaces to create VR content using web technology and can render it using a web browser on VR devices [Mozilla 2013; Vukicevic et al. 2013]. As a result, technical barriers and compatibility issues for creating XR content have been overcome. However, WebVR only deals with a few limited interaction devices compared to the traditional VR environment such as the Virtual-Reality Peripheral Network (VRPN) that provides a unified interface to input devices in VR applications [Taylor II et al. 2001]. Recently, handling user interaction on the web has been proposed [Franke et al. 2012; Olbrich et al. 2013; Redig 2013]. Collaborative work on the web has been focused on concurrent document editing systems such as Google docs [Google 2006], which uses only legacy 2D human computer interaction.

To promote the development of collaborative interaction space for XR applications with various interaction devices on the web, traditional and web developers should be able to handle device events from interaction devices easily using their familiar 
methods. Moreover, a method is also needed to support user interaction and content synchronization in the XR environment, as in existing web collaboration. In this paper, we propose a webizing method for human interface devices and events to provide a collaborative interaction space on Web XR environments. The method provides an event negotiation mechanism to serve different abstraction types (i.e., VRPN messages, X3D sensor data, and HTML DOM events) of interaction events. In addition, we propose a webizing XR interaction space that supports various $\mathrm{XR}$ content and interaction synchronization of multiple users to provide ubiquity, interoperability, and scalability. This paper is extended from the idea of the webizing human interface devices for VR system [Seo et al. 2016]. The remainder of this paper is structured as follows. We present supporting related works on user interaction handling in Section 2 after which we introduce the proposed XR interaction space in Section 3. Then, we explain the prototype implementation in Section 4. Finally, we conclude with a summary and an outlook for future work in Section 5.

\section{RELATED WORK}

It is VR and AR environments that have been studied separately, not XR research. In particular, previous researchers have focused on declarative XR content to create content easily. For content modeling for VR, VRML [VRML Consortium 1997] is the first web based 3D format built using the Silicon Graphics Open Inventor format for describing interactive 3D objects and worlds on the Internet and X3D [X3D 2004] is the successor. XML3D is a declarative approach that describes interactive 3D content as part of a web page [Sons et al. 2010]. The W3C Community Group proposed "Declarative 3D for the Web Architecture" (Dec3D) to add interactive high-level declarative 3D objects as a part of HTML document [W3C Community Group 2013]. Mozilla proposed A-Frame, which is an entity component system web framework for building virtual reality authoring with HTML without build steps [Mozilla 2015]. For AR content, Argon [MacIntyre et al. 2011] uses a KML AR markup language (KARML) for rendering HTML 2D and COLLADA 3D presentation content to physical locations. SmartReality shows how things of interest using semantic web and linked data technologies are incorporated into an AR platform on the Web [Nixon et al. 2012]. ARML 2.0 [Lechner 2013] provides declarative AR content based on XML format. Webized VR and AR content [Seo et al. 2016] proposed web based content authoring using web standards such as HTML, CSS, and Javascript. However, these methods enable VR and AR technologies on the web but do not provide a way to manage interaction space and handle user interaction events.

To handle user interaction events in the traditional VR environment, VRPN comprises a set of classes within a library and servers that provide a device-independent and networktransparent interface between VR applications and physical peripherals such as trackers and buttons [Taylor II, et al. 2001]. Wang and Lindeman [2014] proposed a hybrid virtual environment (HVE) system that combines a tablet device, an
HMD-and-wand- based immersive setup through VRPN, and the unity indie VRPN adapter (UIVA). Redig [2013] developed vrpnwebapp, which is an HTTP server of VRPN server to connect physical devices and web VR applications. The applications receive and handle message streams, but the event handling method on web applications is different from traditional VR native applications. Web applications use HTML DOM events for handling user interactions according to the HTML DOM level 2 events specification [Pixley 2000]. Seo, et al. [2016] proposed a webizing human interface devices for virtual reality that supports VRPN messaging and HTML DOM events according to content negotiation. The W3C Device API [Hirsch 2009] provides a way to access device functions on the web browser and Mozilla's WebVR [Vukicevic, et al. 2013] specifies WebVR related hardware interfaces such as HMD and gamepads. The WebXR Device API [Jones and Waliczek 2017], a successor to WebVR, extends the scope of WebVR into WebXR and specifies general peripheral devices for WebXR on the web. For X3D, Franke, et al. [2012] and Olbrich, et al. [2013] proposed a user interaction input node that extends UserSensor node [Franke et al. 2011]. The X3D specifications include the scene access interface (SAI) API to manipulate the scene during runtime and $\mathrm{X} 3 \mathrm{DOM}$ uses the DOM for this purpose. However, these methods are interfaces to update an X3D scene rather than to handle user interaction.

Second Life [Linden Lab 2003] provides a VR environment for multiple users but does not handle various interaction devices. Davis et al. extended operational transformation to support synchronous collaborative editing applicable to the web [Davis et al. 2002]. Google docs [Google 2006] has enabled multiple users to collaborate on web browsers and ShareDB [ShareDB 2011] provides concurrent editing systems for frontends on the web, while Heinrich et al. proposed the generic transformation approach for multi-user editing [Heinrich et al. 2013; Heinrich et al. 2012]. However, these methods are not suitable for XR environments because they provide content synchronization in an existing web environment only and do not support the handling of various interaction devices in web XR environments.

\section{WEBIZING XR INTERACTION SPACE}

For multiple users to interact when collaborating, a collaborative interaction space should be easily accessible and should synchronize XR content according to user interaction results in real time. The specific design goals of the collaborative interaction space are as follows:

- Ubiquity: the collaborative interaction space for multiple users is easily accessible at any time and from anywhere and provides a unique addressing mechanism to access the space.

- Interoperability: user interaction events should be represented using standards and handled regardless of XR content description methods.

- Scalability: the interaction space should support various types of interaction devices and should provide 
participation of multiple users. In addition, it should be possible to manage multiple interaction spaces.

To satisfy these requirements, we propose a webizing $\mathrm{XR}$ interaction space method that deals with user interaction events using web technologies and supports collaborative interaction by multiple users.

\subsection{Webizing Human Interface Devices}

Users generate interaction events using various interaction devices, and these events are processed according to their XR content through the webizing process. Figure 1 shows an overview of our proposed webizing human interface devices for a single user.

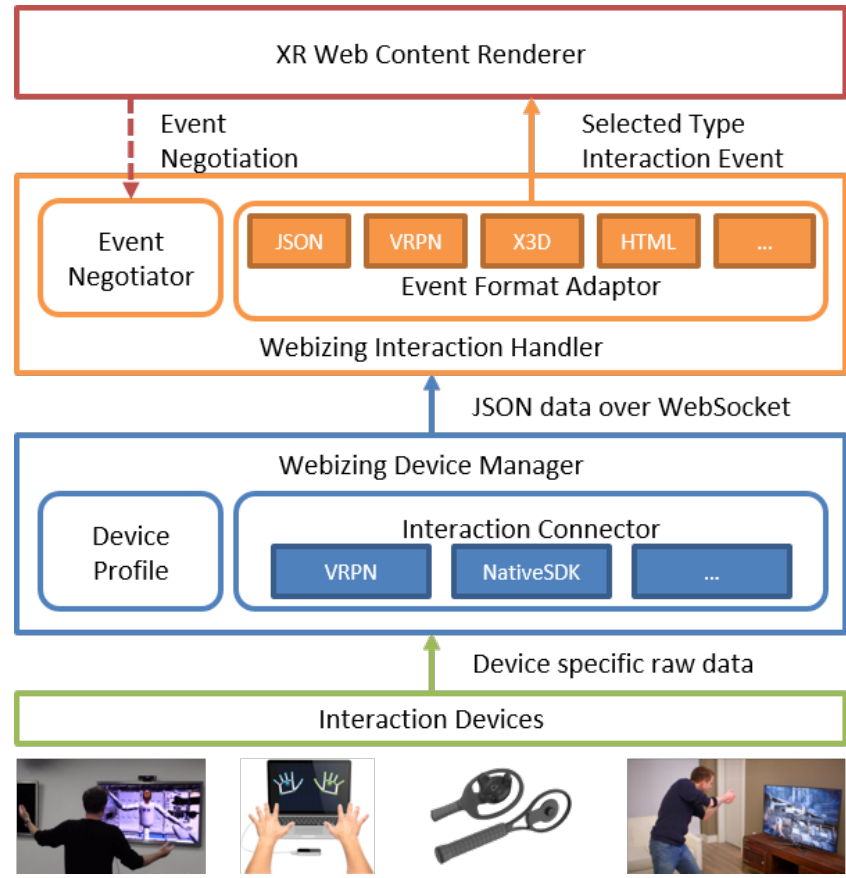

Figure 1: An overview of webizing human interface devices

The user generates an event using suitable interaction devices and the event is delivered to the webizing device manager. Each interaction device has its own device profile that contains a device identification, interaction event types, and device status, as shown in Listing 1.

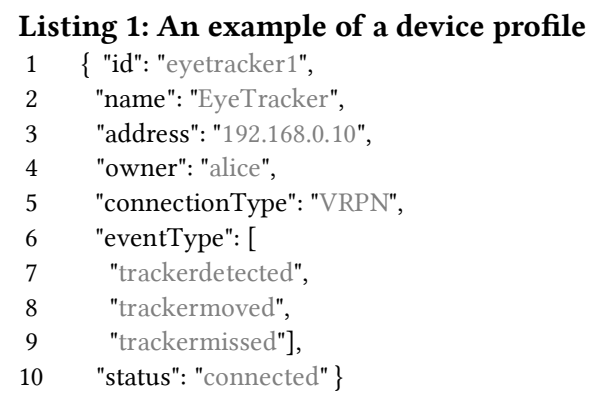

The interaction connector is an interface for tracker, button, analog, and dial input devices, among others. Tracker devices such as OptiTrack, Leap Motion, Tobii's eye tracker, Intel's RealSense, and AR marker tracker report the pose (i.e. position and orientation), velocity, and acceleration of targets, while button devices such as a gamepad or keyboard send the press and release status of one or more buttons. Analog devices such as an analog stick or joystick notify one or more analog values and the dial devices such as Microsoft's Surface Dial report incremental rotation. Device specific raw data from interaction devices are delivered to a common interface in the interaction connector. Developers can choose the connecting method between input devices and interaction connectors such as VRPN or device specific interfaces provided by manufacturers, including native device drivers and software development kits (SDKs).

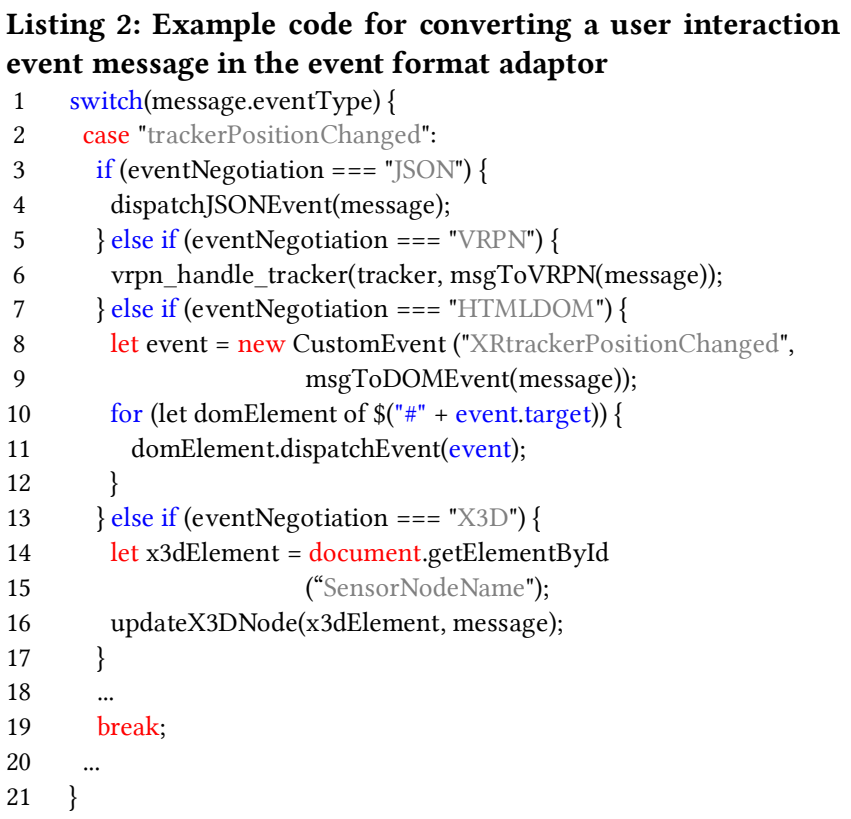

The device specific raw data are converted to JSON format in the interaction connector and passed on to the webizing interaction handler working on the web browser. The handler deals with event negotiation, which is a mechanism to provide the same events from different types of user interaction event (i.e. VRPN messages, X3D sensor data, or HTML DOM events). Event negotiation is a similar operation to HTTP content negotiation [Fielding et al. 1999]. The event handling adapter in the interaction handler is determined according to the event negotiation result. The event format adaptor converts JSON formatted data delivered from the interaction connector. The XR web content renderer is implemented by web technologies and it uses JavaScript to handle user interaction events. The XR web content renderer visualizes XR content such as X3D or HTML documents on the $3 \mathrm{D}$ web. When the XR web content renderer is loading, the global variable eventNegotiation is used for event negotiation by the event negotiator. The developers can 
implement the XR web content renderer using a familiar method to handle user interaction events. Listing 2 shows an example of the event format adaptor that converts the user interaction events according to the event negotiation and invokes a function to deliver the interaction event. Developers can choose the type of interaction event to extend the event format adapter.

Listing 3(a) shows an example of a VPRN message. To process one, a developer declares the device being used as a VRPN message in his/her code and handles it according to the device type. Listing 3(b) is an HTML DOM event example for which a developer registers a listener for each type of interaction event and the listener processes the event when a user triggers the type of event.

Listing 3: Event handling example from a tracker device on a web browser: (a) a VRPN message handling example and (b) an HTML DOM event handling example

1 let tracker = new Webized_VRPN_Tracker("Tracker0@localhost");

2 function vrpn_handle_tracker(tracker, data) \{

3 // do something

$4\}$

1 let domElement = document.getElementById("element_id");

2 domElement.addEventListener("XRtrackerPositionChanged",

trackerPosHandler);

function trackerPosHandler(event) \{ // do something \}

\subsection{Webizing Collaborative Interaction Space}

We designed a collaborative interaction space using web technologies to support ubiquity, interoperability, and scalability for collaboration of multiple users. The interaction space is identified by URI and a user can access the space on the web browser. Interaction events are handled by the webizing human interface devices method given in Figure 1. The interaction events are described by JSON formatted data and are exchanged using web standards.

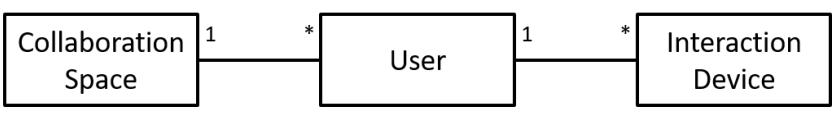

Figure 2: Relationship among collaboration space, user, and interaction device

The relationship among the interaction space, user, and interaction device is shown in Figure 2. Each user can use multiple interaction devices and multiple users can participate in one collaborative space. Collaborative spaces are created or destroyed according to the user request, and XR content that describes a collaboration space is updated by user interactions and stored. The relationship between a user and the user's interaction devices are changed by his/her selection in the collaboration space. For example, Alice has three kinds of interaction devices such as Leap Motion, a keyboard, and a mouse. Bob has two types of interaction devices such as
RealSense and a mouse. Alice creates an XR space for collaborative work and shares the space with Bob, after which Alice and Bob choose interaction devices to use in the XR space. Alice selects a Leap Motion device and Bob selects all of his devices. In this example, the relationship between the collaboration space and users is one-to-two. The relationship between Alice and her devices is changed to one-to-one in the current XR space and the relationship between Bob and his devices is change to one-to-two in the current XR space.

An overview of the proposed system is shown in Figure 3, which demonstrates how to deal with multiple user interactions in a collaborative interaction space of an XR environment. Handling individual user's interaction events and rendering XR content is processed in the XR web client, and to deliver these to other users, the event handler sends and receives JSON formatted interaction event data through the XR interaction space server. The webizing interaction handler deals with the other users' received interaction data as if it was self-interaction data. The event handler also deals with content modification and synchronization events. The XR interaction space server manages the XR content cycle and delivers user interaction events among the users in the collaborative interaction space. The Content DB in the server stores the XR content representing the XR space, user authentication and authority information, and $\mathrm{XR}$ space status. The space manager has a role in routing user interaction events and managing the content and users. To deliver user interaction events, the event router in the space manager receives and sends user interaction data and content related events using the WebSocket protocol. The content manager in the space manager deals with user requests from the RESTful API to update the XR content and XR space status information. The auth manager provides access control for each XR collaborative interaction space. The server handles arbitrary web content because the server design is content independent and follows web standards.

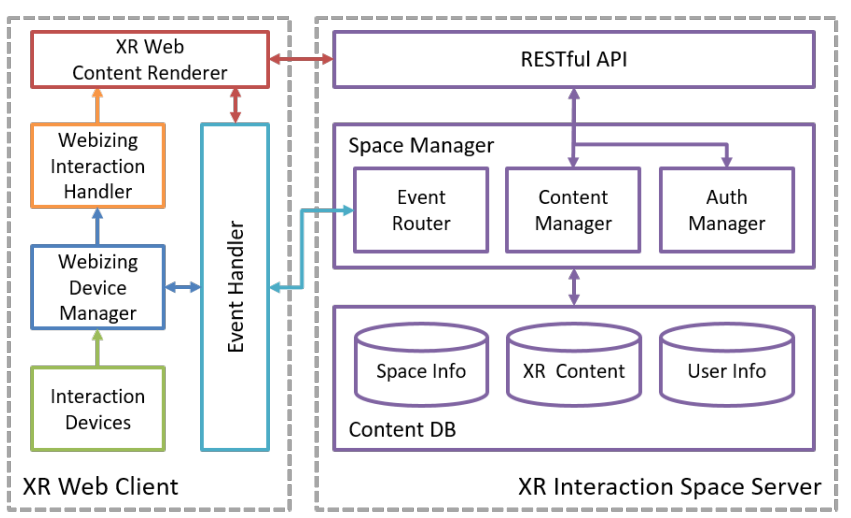

Figure 3: Overview of a webizing collaborative interaction space

\section{IMPLEMENTATION}

We implemented various prototype applications using human interface devices and a collaboration space. The experiment was 
performed using Intel's i7-7700k CPU and NVIDIA's GTX 1080 GPU on Google Chrome web browser version 65 (64-bit). The XR interaction space server is implemented by node.js and uses deepstream.io [deepstreamHub 2015] for the event router. The deepstream.io is a WebSocket server for data synchronization between browsers using a lightweight way of publish-subscribe pattern in real-time. We use Intel's RealSense and Leap Motion devices for hand gestures.

\subsection{Webizing Single User Interaction}

Figure 4 shows an example application of a single user interaction using a single webizing human interface device to control a flight vehicle. The interaction device recognizes the movement of the user's head, as shown in Figure 4(a). To use the interaction device, a user declares a device profile of Intel's RealSense as a tracker device. The tracker device receives raw data using the native SDK in the webizing device manager (Figure 1). The webizing device manager delivers the raw data to the webizing interaction handler using the WebSocket protocol. When the required interaction devices for the application have been matched, the application is started. The webizing device manager returns the status of the interaction device and the application recognizes the available user interaction devices.
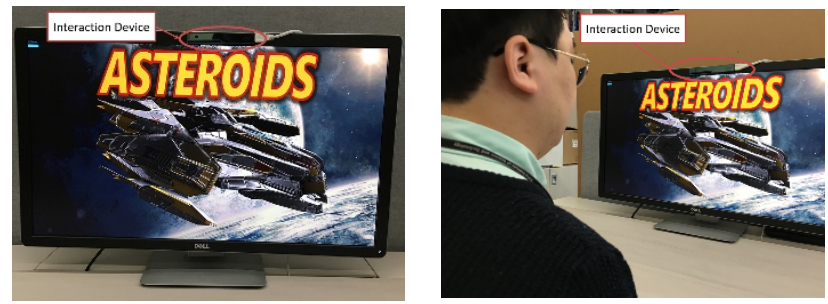

(a)
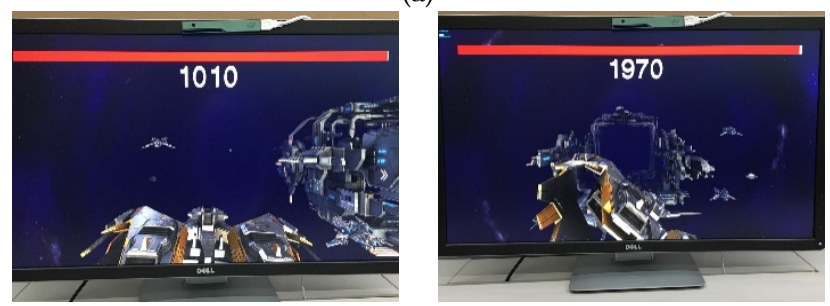

(b)

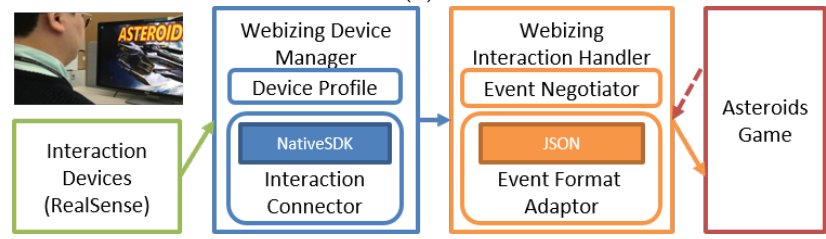

(c)

Figure 4: Experimental results of webizing the peripheral for a single user: (a) the webizing device for the 3D web game, (b) the user's view on the web browser, and (c) an overview of webizing the single user interaction space

Figure 4(b) shows a user's perspective of the application. When the application is starting, it performs an event negotiation. The application sends a requested event type to the webizing interaction handler and then receives an interaction event based on the event negotiation result. The application uses a JSON formatted interaction event and the flight vehicle avoids obstacles in the application. The RealSense interaction device tracks the three-dimensional (3D) user's head position and sends the position to the webizing interaction handler, from which the application receives the position data and updates the XR content according to the user interaction. Figure 4(c) describes the event handling process of a user interaction in this application.

\subsection{Webizing Collaborative Interaction Space for Multiple Users}

To support multiple users in a collaboration interaction space, a user registers his/her interaction devices on the XR interaction space server. Each user in the collaboration interaction space uses the XR web client (as shown in Figure 3) via the same process as for a single user interaction. To join the interaction space, a user logs in to the XR interaction space server and shares the URI of the participated in space. The web page shows the user's own registered interaction devices (the relationship is shown in Figure 2), for which the user can add, update, or delete profiles. The availability of each XR interaction space is shown according to the user's interaction devices and authorities.

We modified the Jenga example from Physijs [Prall 2012] to use it as a collaborative interaction game, as shown in Figure 5. The game is a turn-based classic block-stacking and stackcrashing game. The modified example uses hand interaction to pick up and move a block using a Leap Motion controller, which is a hand tracking interaction device; any type of hand tracking device is acceptable for this game. Figure 5(a) shows the configuration for each participant in the collaborative interaction game. The Leap Motion controller is connected to a desktop computer by a USB cable, and interaction events from the device are collected using the SDK provided by the product manufacturer. Two users, Bob and Dave, play the Jenga game. They wear a mobile HMD VR device and the game works on the web browser of the mobile VR device. They register the device profile of each Leap Motion device to the webizing device manager. The webizing interaction handler can chooses the interaction device based on the device profile. When the game is started, it checks the connectivity and availability of the hand tracking device. If the game cannot find one, it shows a guide message to check the device status and waits for device connection.

The game executes event negotiation to choose a type of event handling process and the HTML DOM event is selected in this game. The game converts raw interaction events to HTML DOM events and deals with the interaction HTML DOM events. Each webizing device manager receives raw data from the connected Leap Motion device and converts it to JSON data. When the Jenga game is started, Bob's hand on the screen is updated continuously and the interaction event of the hand is delivered to Dave. The webizing interaction handler exchanges the JSON data with the other users and generates an HTML DOM event according to the user's own and received JSON data. 
The webizing interaction handler distinguishes the interaction events based on the owner attribute in the device profile, as shown in Listing 1 and the event type, as shown in Listing 2. The Figure 5(b) is a Bob's view. Bob's hand is rendered in blue located in bottom-left and Dave's user's hand in red located in bottom-right. When Bob's turn, he can pick up and move blocks on the screen using the hand as shown in Figure 5(b). If it is not the Bob's turn, he cannot pick up and move blocks. Figure 5(c) shows the connection status of the interaction devices and a handing process overview of the game.

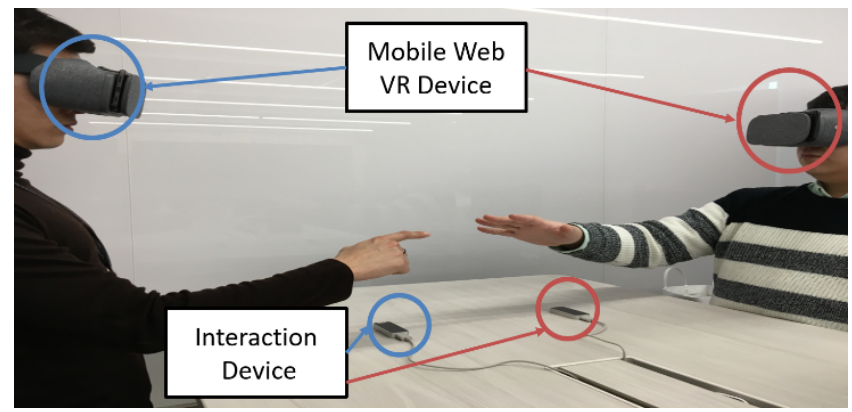

(a)

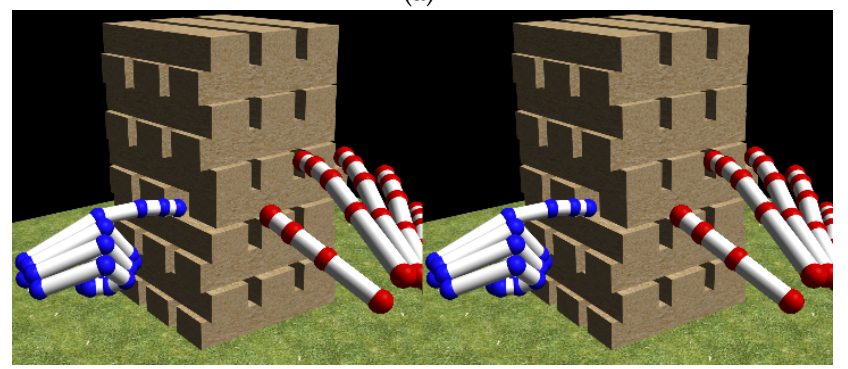

(b)

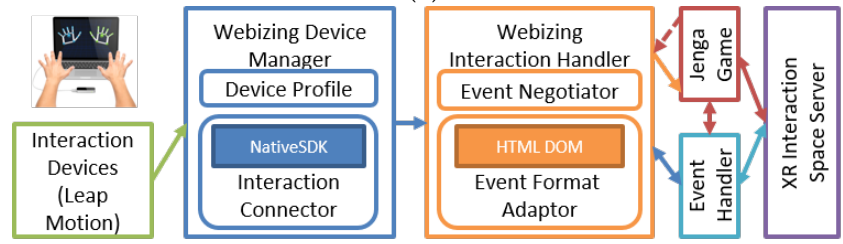

(c)

Figure 5: Experimental results of webizing the peripherals for multiple users in collaboration space: (a) the webizing interaction devices for the mobile web application, (b) the users' view on the web browser using mobile HMD VR device, and (c) an overview of webizing the collaborative interaction space

Figure 6 shows an example of multiple user interactions with multiple devices. In this example, two users review a $3 \mathrm{D} C \mathrm{CAD}$ model on a web browser concurrently. The 3D CAD model is declared by A-Frame [Mozilla 2015] in an HTML document and user interaction events are processed using VRPN messages as shown in Listing 2(b). The webizing device manager deals with Leap Motion and mouse devices. The user uses hand gestures to select a menu item or change viewpoint, as shown in Figure 6(a) and uses a mouse for precise work such as the transformation of the $\mathrm{CAD}$ model as shown in Figure 6(b). The webizing interaction handler exchanges event data from interaction devices and generates VRPN messages. In this example, the content and viewpoint are synchronized concurrently whereas interactions such as selecting a menu are not.

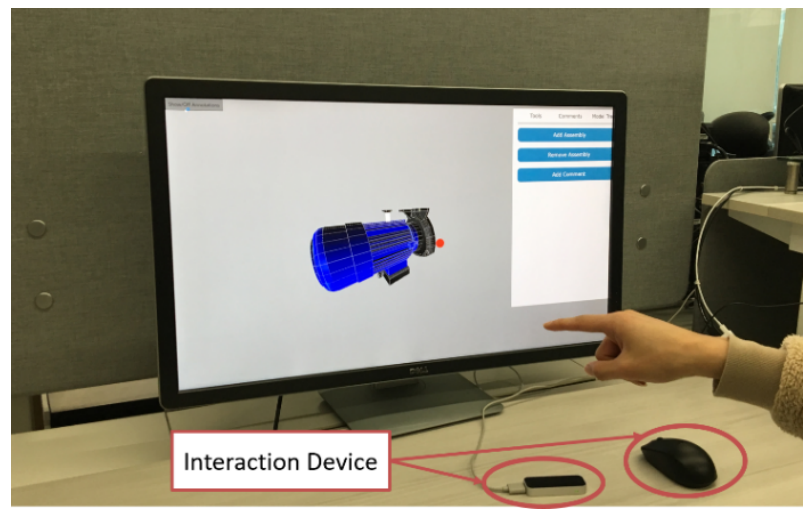

(a)

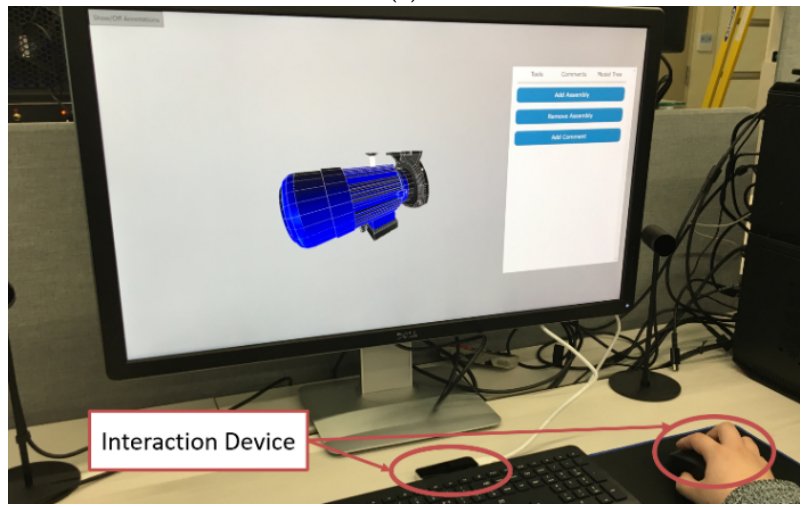

(b)
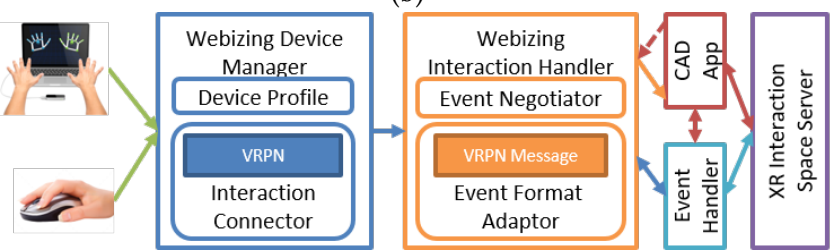

(c)

Figure 6: Content synchronization example of multiple users with multiple devices in real-time: (a) a hand gesture to change the viewpoint, (b) changing the scale of a $3 \mathrm{D}$ CAD model using a mouse, and (c) an overview of webizing the collaborative interaction space for $3 \mathrm{D} C A D$

\subsection{Webizing Collaboration for Cross Reality}

Figure 7 is a collaboration example to maintain a chamber in various XR environments. A worker uses a VR HMD device for a chamber maintenance training application and an AR browser to fix a real chamber. An expert uses a 3D web application to write the maintenance manual for the chamber, and the VR application and remote AR browser are utilized to guide the worker. 


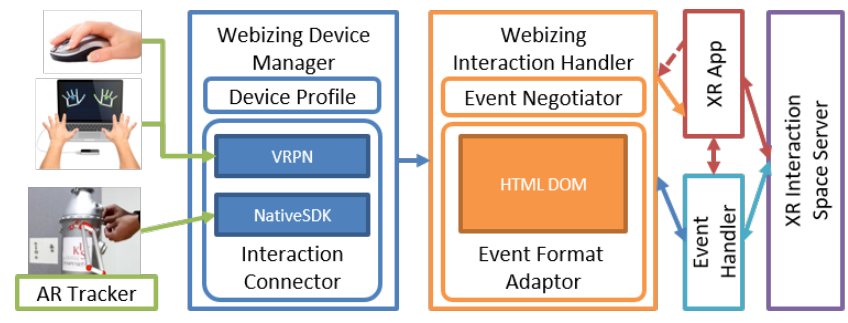

Figure 7: An overview of an XR collaboration space with multiple interaction devices

Figure 8 to Figure 10 show the detailed processes of a worker and an expert. The expert chooses a 3D web environment and creates a manual for chamber maintenance in it, as shown in Figure 8. The application gives a URI for the XR space that is shared with the worker for VR training and AR maintenance. The expert uses a keyboard and mouse device and adds device profiles for them, then arranges multimedia tutoring materials around the chamber $\mathrm{CAD}$ model with the maintenance instructions. The webizing interaction handler deals with user interaction events according to the event negotiation result. In this example, the application uses an HTML DOM event type.

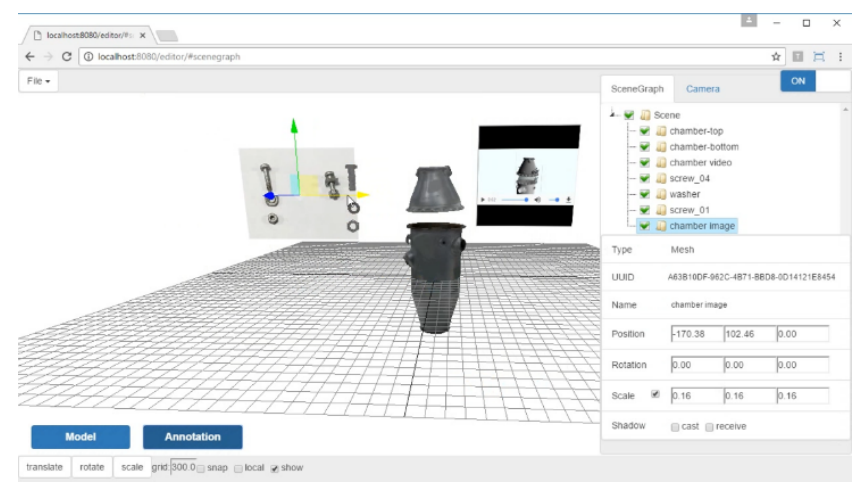

Figure 8: A 3D mode example of creating a manual for chamber maintenance in XR environments

Using this manual, the worker performs the chamber repair training. Figure 9 shows an example of collaboration training between the worker and expert in the VR environment. The worker opens the collaboration space URI shared by the expert. The worker chooses a Leap Motion device for hand gesturing and registers the device profiles to the webizing device manager. $\mathrm{He} / \mathrm{she}$ wears an HMD device and practices the training with the interaction device to recognize hand gestures, as shown in Figure 9(a). The application checks the device connection status and executes event negotiation to deal with interaction events using the webizing interaction handler. The expert also registers his/her Leap Motion device for hand gesturing. The expert accesses the space on a web browser using the shared URI. As the worker performs training, the expert's view is also updated in real time. When the worker performs a wrong instruction, the expert gives the right one using his/her interaction device to capture his/her hand gestures as shown in Figure 9(b). The hand gesture events of the worker and expert are shared using the webizing interaction handler.

Figure 10 is an example of remote collaboration in an XR environment. Figure 10(a) shows the repair process of a real chamber in an AR environment. The worker uses a web based AR browser to check the manual, as exhibited in Figure 8 . The URI of the collaborative XR space is shared with the expert who can view a remote video stream of the worker's AR browser in real time, as shown in Figure 10(b). The expert uses an interaction device for hand gestures to instruct using hand gesturing within the collaboration space, as shown in Figure 9, and the instructions are shared and processed using the webizing interaction handler.

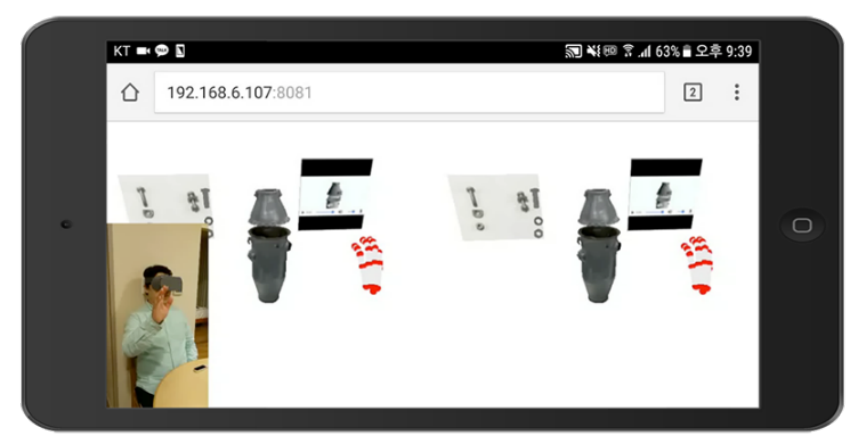

(a)

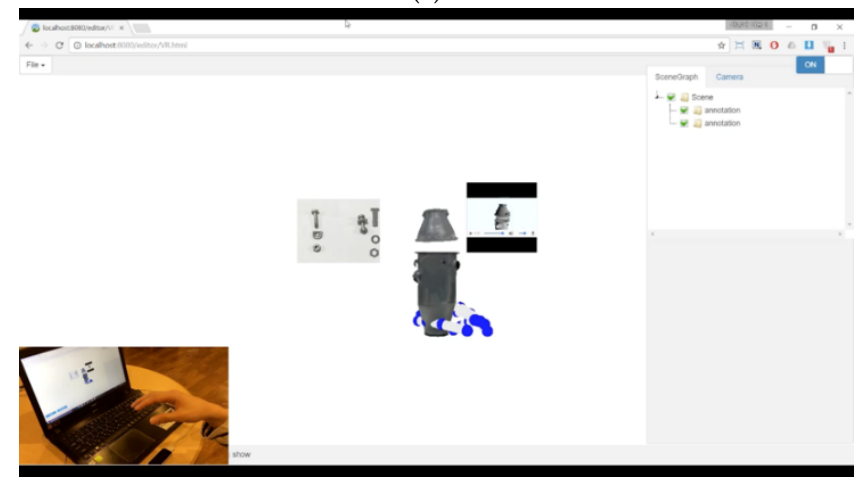

(b)

Figure 9: An example of collaboration for training: (a) the worker in VR mode with an HMD device and (b) the expert in $3 \mathrm{D}$ mode on a web browser

Through these prototype examples, we have shown that our proposed method can be used for interaction devices on the web regardless of the interface type, which makes it easy to access an interaction space using a URI on a web browser. It also shows that multiple users can use multiple interaction devices in the interaction space for collaboration purposes and the management of the interaction space is scalable.

\section{CONCLUSION}

In this paper, we propose webizing human interface devices and collaborative interaction spaces for XR in a web environment. We use an event negotiation mechanism to provide different 
abstraction types of user interaction events. As a result, developers can choose a familiar way to implement XR web applications and use resources and libraries on the web. In addition, the developers can use our proposed webizing XR interaction space for synchronization of XR content and interaction events among multiple users. We expect that our proposed method will promote the development of XR web applications with various interaction devices through traditional $\mathrm{XR}$ and web development.

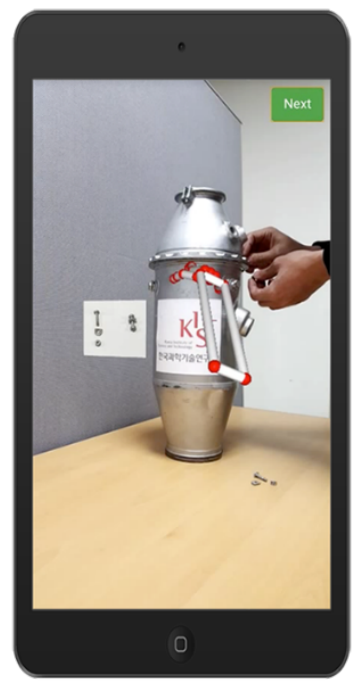

(a)

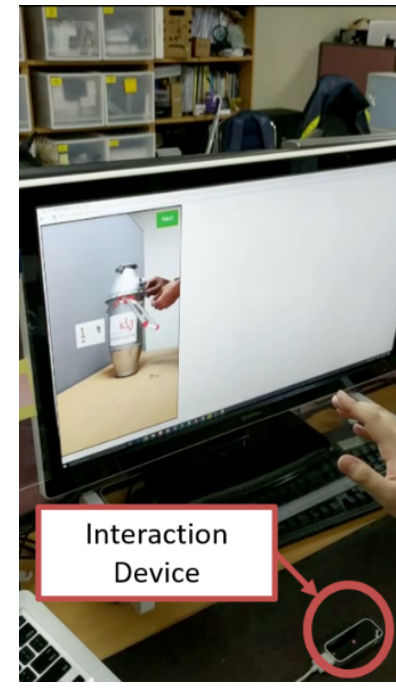

(b)
Figure 10: Remote collaboration in an XR environment: (a) the worker fixes a chamber in AR mode and (b) the expert gives instructions to the worker using hand gesturing via remote video on a web browser

\section{ACKNOWLEDGMENTS}

This work was supported by the National Research Council of Science \& Technology (NST) grant by the Korea government (MSIT) (No. CMP-16-01-KIST).

\section{REFERENCES}

Davis, A.H., Sun, C. and Lu, J. 2002. Generalizing operational transformation to the standard general markup language. In Proceedings of the ACM conference on Computer supported cooperative work, New Orleans, LA, USA, Nov 16-20 2002 ACM, 587088, 58-67.

deepstreamHub, 2015. deepstream.io [online]. https://deepstreamhub.com/opensource/?io [Accessed Apr 13 2018]

Dionisio, J.D.N., III, W.G.B. and Gilbert, R. 2013. 3D Virtual worlds and the metaverse: Current status and future possibilities. ACM Computing Surveys 45, $1-38$.

Fielding, R., Gettys, J.J., Nielsen, J.M.H.F., Masinter, L., Leach, P. and Berners-Lee, T. 1999. Content Negotiation of Hypertext Transfer Protocol -- HTTP/1.1.

Franke, T., Kahn, S., Olbrich, M. and Jung, Y. 2011. Enhancing realism of mixed reality applications through real-time depth-imaging devices in X3D. In Proceedings of the International Conference on 3D Web Technology, Paris, France, Jun 20-22 2011 ACM, 2010439, 71-79.

Franke, T., Olbrich, M. and Fellner, D.W. 2012. A flexible approach to gesture recognition and interaction in X3D. In Proceedings of the International Conference on 3D Web Technology, Los Angeles, CA, USA, Aug 4-5 2012 ACM, 2338743, 171-174.

Google, 2006. Google Docs [online]. https://www.google.com/docs/about [Accessed Jan 31 2018].

Heinrich, M., Lehmann, F., Grüneberger, F.J., Springer, T. and Gaedke, M. 2013 Analyzing the suitability of web applications for a single-user to multi-user transformation. In Proceedings of the International Conference on World Wide Web, Rio de Janeiro, Brazil, May 13-17 2013 ACM, 2487914, 249-252.

Heinrich, M., Lehmann, F., Springer, T. and Gaedke, M. 2012. Exploiting single-user web applications for shared editing: a generic transformation approach. In Proceedings of the International conference on World Wide Web, Lyon, France, Apr 16-20 2012 ACM, 2187978, 1057-1066.

Hirsch, F. 2009. Device and Sensors Working Group World Wide Web Consortium. Jones, B. and Waliczek, N. 2017. WebXR Device API - Editor's Draft, 12 December 2017 World Wide Web Consortium.

Lechner, M. 2013. OGC Augmented Reality Markup Language 2.0 (ARML 2.0) Open Geospatial Consortium.

Linden Lab, 2003. Second Life [online]. http://secondlife.com [Accessed Oct 19 2017].

MacIntyre, B., Hill, A., Rouzati, H., Gandy, M. and Davidson, B. 2011. The Argon AR Web Browser and standards-based AR application environment. In Proceedings of the International Symposium on Mixed and Augmented Reality, Basel, Switzerland, Oct 26-29 2011 IEEE, 65-74.

Mozilla, 2013. WebVR [online]. https://webvr.info [Accessed Jun 23 2017].

Mozilla, 2015. A-Frame [online]. https://aframe.io [Accessed Jun 23 2017].

Nixon, L., Grubert, J., Reitmayr, G. and Scicluna, J. 2012. SmartReality: Integrating the Web into Augmented Reality. In Proceedings of the International Conference on Semantic Systems, Graz, Austria, Sep 5-7 2012, 48-54.

Olbrich, M., Franke, T., Keil, J. and Hertling, S. 2013. Skeletal input for user interaction in X3D. In Proceedings of the International Conference on 3D Web Technology, San Sebastian, Spain, Jun 20-22 2013 ACM, 2466559, 143-146.

Pixley, T. 2000. Document Object Model (DOM) Level 2 Events Specification World Wide Web Consortium.

Prall, C., 2012. Physijs: Physics plugin for Three.js [online]. https://github.com/chandlerprall/Physijs [Accessed Jun 10 2016].

Redig, S., 2013. VRPN-webapp: A VRPN server and http server for webapp controlers [online]. https://github.com/Laremere/vrpn-webapp [Accessed Jun 10 2016].

Seo, D., Kim, D., Yoo, B. and Ko, H. 2016. Webizing human interface devices for virtual reality. In Proceedings of the ACM Conference on Virtual Reality Software and Technology, Munich, Germany, Nov 2-4 2016 ACM, 2996307, 369371.

Seo, D., Yoo, B. and Ko, H. 2016. Webizing Mixed Reality for Cooperative Augmentation of Life Experience. In Proceedings of the ACM Conference on Computer Supported Cooperative Work and Social Computing Companion, San Francisco, CA, USA, Feb 27-Mar 32016 ACM, 401-404.

ShareDB, 2011. ShareDB: A database frontend for concurrent editing systems [online]. https://github.com/share/sharedb [Accessed Jan 31 2018].

Somasegar, S., 2017. XR is a new way to consider the reality continuum [online]. https://techcrunch.com/2017/05/02/xr-a-new-way-to-consider-the-realitycontinuum [Accessed Feb 15 2018].

Sons, K., Klein, F., Rubinstein, D., Byelozyorov, S. and Slusallek, P. 2010. XML3D: interactive 3D graphics for the web. In Proceedings of the International Conference on Web 3D Technology, Los Angeles, California, USA, Jul 24-25 2010 ACM, 175-184.

Taylor II, R.M., Hudson, T.C., Seeger, A., Weber, H., Juliano, J. and Helser, A.T. 2001. VRPN: a device-independent, network-transparent VR peripheral system. In Proceedings of the ACM symposium on Virtual reality software and technology, Baniff, AB, Canada, Nov 15-17 2001 ACM, 55-61.

VRML Consortium 1997. ISO/IEC 14772-1:1997 Virtual Reality Modeling Language (VRML).

Vukicevic, V., Jones, B., Gilbert, K., Wiemeersch, C.V., Waliczek, N. and Cintron, R. 2013. WebVR World Wide Web Consortium.

W3C Community Group, 2013. Declarative 3D for the Web Architecture [online]. https://www.w3.org/community/declarative3d [Accessed Apr 7 2016].

Wang, J. and Lindeman, R. 2014. Coordinated 3D interaction in tablet- and HMDbased hybrid virtual environments. In Proceedings of the ACM symposium on Spatial user interaction, Honolulu, HI, USA, Oct 4-5 2014 ACM, 70-79.

Wikipedia, 2017. X Reality (XR) https://en.wikipedia.org/wiki/X_Reality_(XR) [Accessed Feb 15 2018].

X3D 2004. ISO/IEC 19775:2004 Extensible 3D (X3D). 